\title{
Convergence Control for Topology Optimization
}

\author{
Dylan Bender and Ahmad Barari \\ Faculty of Engineering and Applied Science \\ The University of Ontario Institute of Technology \\ Oshawa, Canada \\ Ahmad.Barari@uoit.ca
}

\begin{abstract}
Proportional-Integral-Derivative (PID) control theory is applied to the evolutionary rate of the Bi-Directional Evolutionary Structural Optimization (BESO) method to control aspects of the convergence such as the rise time, stability, and other convergence characteristics. When the PID controller is applied to the BESO topology optimization method, its behavior resembles that of a second order linear system and its response depends on whether it is an overdamped, critically damped or underdamped system. The new algorithm replaces the evolutionary rate control parameter with the three gain values of the controller, namely, the proportional gain, the integral gain and the derivative gain for further control the structure's evolution.
\end{abstract}

Keywords - topology optimization; adaptive optimization; Proportional-Integral-Derivative; Bi-Directional Evolutionary Structural Optimization ; PID control

\section{INTRODUCTION}

Structural optimization consists of three main categories, which are size, shape and topology optimization. Size optimization aims to find an optimal design by optimizing variables such as cross-sectional dimensions or thicknesses. Shape optimization achieves structural optimization by changing pre-determined boundaries. Topology optimization, the concern of this paper, aims to determine the best locations and geometries of cavities within the design domain [1]. Fig. 1 shows a sample problem definition and resulting optimal structure of the Bi-Directional Evolutionary Structural Optimization method of topology optimization (BESO).. Although the resulting structure has much less material and weight, but it maintain the required mechanical stiffness for the given loading and boundary condition. Reducing weigh of the components is highly desired in various applications, particularly in design and manufacturing of automotive and aerospace products.

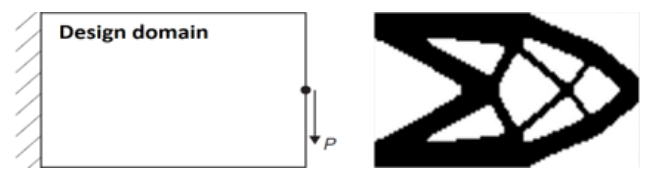

Figure 1. Sample topology optimization problem definition (left) and resulting structure (right)

Significant developments have been seen in topology optimization methodologies within the last two decades [2], the majority of which are based of Finite Element Analysis. However, applications of the developed topology optimization methods have been very limited due (i) the limitations in manufacturing and inspection of the complex topologies, and (ii) the computational complexity of the topology optimization algorithms. A successful design process needs to be conducted by considering the details of the manufacturing and inspection requirement [3]. Although the additive manufacturing processes are still not able to produce high surface qualities [4], they are highly flexible in producing the complex geometries resulting by the topology optimization methodologies [5]. Also. The advances in coordinate metrology algorithms allow relatively fast inspection of the complex topologies resulting by the topologically optimized designs [6].

The computational complexity is another limiting factor in implementation of the topology optimization algorithms. There is not that much control on the convergence of these algorithms and their computational efficiencies are not addressed well. This paper will focus on the convergence control of a typical topology optimization algorithm, Bi-directional Evolutionary Structural Optimization. BESO first introduced by [7]. The BESO method contains several control parameters including target volume fraction, penalty, minimum density, filter radius, mesh resolution, and the focus of this paper, the evolutionary rate. Traditionally, the evolutionary rate is taken to be a constant value, usually $1 \%, 2 \%$ or even as high as $4 \%$, but there exists potential to use the evolutionary rate as a parameter of adaptive optimization in the form of a Proportional-IntegralDerivative controller in conjunction with closed-loop feedback.

Feedback is a very powerful idea. Its use has often had revolutionary consequences with drastic improvements in performance [8]. The usefulness of PID controls lies in their 
general applicability to most control systems. In particular, when the mathematical model of the plant is not known and therefore analytical design methods cannot be used, PID controls prove to be most useful [9]. For this paper, the volumetric error signal is used to control the evolutionary rate of the BESO algorithm. The volumetric error is calculated as the difference in the targeted volume fraction and the current iterations volume fraction which can then be integrated and differentiated to produce the three inputs of the PID controller.

\section{BACKGROUND}

\section{A. Bi-directional Evolutionary Structural Optimization}

In the Bi-Directional Evolutionary Structural Optimization (BESO) method, the optimal topology is determined according to the relative ranking of the elemental sensitivity numbers. The sensitivity numbers of the elements are conceptualized to be the increase in the mean compliance, $\mathrm{C}$, of a structure as a result of the removal of the ith element is equal to its elemental strain energy [1]. By this definition, the optimization problem is to find the most effective way to remove elements so that the increase in the compliance is minimal as shown in (1).

Minimize:

$$
C=\frac{1}{2} f^{T} u
$$

Where $\mathbf{f}$ is the nodal DOF force vector and $\mathbf{u}$ is the nodal displacement vector, both of which has the size $2 *(\text { nely }+1)^{*}($ nel $x+1)$ where nelx and nely are the number of elements in the $\mathrm{x}$ - and $\mathrm{y}$-dimensions respectively of the finite element mesh.

The study on the effect of element removal on the stiffness is referred to as the sensitivity analysis. Sensitivity numbers, which indicate the change in the overall stiffness (strain energy), have been formulated using information available from a static finite element analysis [10]. The sensitivity number of the ith element is calculated as:

$$
\frac{\partial C}{\partial x_{i}}=-\frac{1}{2} p x_{i}^{p-1} u_{i}^{T} K_{i}^{0} u_{i}
$$

Each iteration of the topology optimization problem is subject to equation (3) and (4) where $V^{*}$ is the prescribed target volume, $V_{i}$ and $x_{i}$ are the volume and density of each element respectively and $\mathrm{N}$ is the total number of elements.

$$
\begin{gathered}
V^{*}-\sum_{i=1}^{N} V_{i} x_{i}=0 \\
x_{i}=x_{\min } \text { or } 1
\end{gathered}
$$

The target volume for the subsequent iteration $\left(\mathrm{V}_{\mathrm{i}+1}\right)$ or $\left(V^{*}\right)$ is determined based on the current structural volume, $V_{i}$ the target structural volume, $\mathrm{V}_{\mathrm{c}}$, and the main focus of this paper, the evolutionary rate, ER. The subsequent target volume is calculated as follows:

$$
V_{i+1}=\left\{\begin{array}{l}
\max \left(V_{i}(1-E R)_{v} V_{c}\right) \text { when } V_{i}>V_{c} \\
\max \left(V_{i}(1+E R)_{0} V_{c}\right) \text { when } V_{i} \leq V_{c}
\end{array}\right.
$$

Convergence Criterion:

$$
\text { error }=\frac{\left|\sum_{i=1}^{W} C_{k-i+1}-\sum_{i=1}^{W} C_{k-N-i+1}\right|}{\sum_{i=1}^{W} C_{k-1+1}} \leq \tau
$$

The soft-kill BESO MATLAB code downloaded from the website www.isg.rmit.edu.au is used as a test platform for the methods proposed in this paper. The design domain is assumed to be rectangular and discretized using four node plane stress elements. Below, in fig. 2, a short cantilever is taken as an example of the resulting structure of the unmodified code (the graph was generated by the authors of this paper) with the input data being: nelx $=120$, nely $=60$, volfrac $=0.5, E R=0.02$, and rmin $=3$.

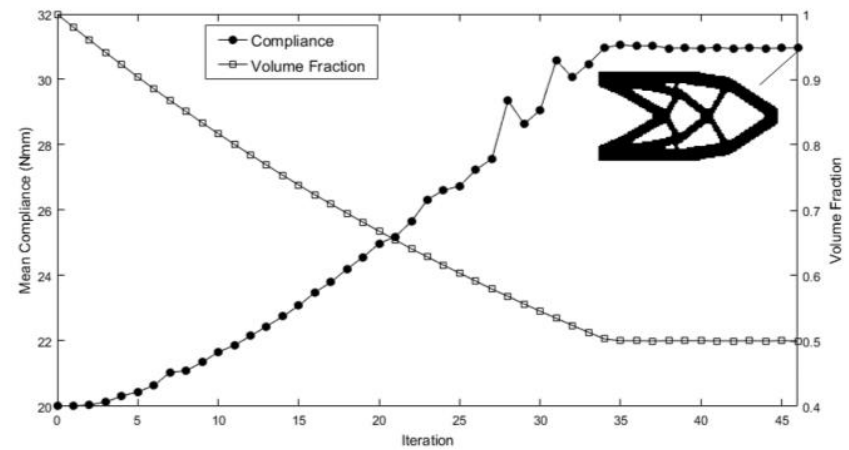

Figure 2: Results of a topology optimized short cantilever with optimization parameters: nelx $=120$, nely $=60$, volfrac $=0.5, \mathrm{ER}=0.02$, rmin $=3, \mathrm{p}=3$. Resulting compliance: $30.96 \mathrm{Nmm}$.

\section{B. PID Control of a Second Order Linear System}

A standard Proportional-Integral-Derivative controller, or PID controller for short is a form of a "three-term" controller whose transfer function is wrtten as:

$$
G(s)=K_{P}+K_{I} \frac{1}{s}+K_{D} s
$$

The "three-term" functionalities are the proportianl term, the integral term, and the derivative term. The proportional term provides an overall control action proportional to ther error signal through the proportional gain factor. The integral term reduces steady-state errors through low-frequency compensation by an integrator and the derivative term improves the transient response through high-frequency compensation via a differentiator [8]. The effects of increasing these terms can be seen below in Table 1. Rise time refers to the number of iterations to reach the target volume for the first time, overshoot is the maximum peak value of the response curve (volumetric error curve) measured from target volume. Settling time refers the number of iterations for the response curve to reach and stay within a range of the final volume fraction. Steady state error refers to the difference between the final equallibrium and the target volume of the response curve. 
Table 1. Effects of independent P, I, and D tuning [7]

\begin{tabular}{|l|l|l|l|l|l|}
\hline $\begin{array}{l}\text { Closed- } \\
\text { Loop } \\
\text { Response: }\end{array}$ & $\begin{array}{l}\text { Rise } \\
\text { Time }\end{array}$ & Overshoot & $\begin{array}{l}\text { Settling } \\
\text { Time }\end{array}$ & $\begin{array}{l}\text { Steady } \\
\text { State } \\
\text { Error }\end{array}$ & Stability \\
\hline $\begin{array}{l}\text { Increasing } \\
\mathrm{K}_{\mathrm{P}}\end{array}$ & Decrease & Increase & $\begin{array}{l}\text { Small } \\
\text { Increase }\end{array}$ & Decrease & Degrade \\
\hline $\begin{array}{l}\text { Increasing } \\
\mathrm{K}_{\mathrm{I}}\end{array}$ & $\begin{array}{l}\text { Small } \\
\text { Decrease }\end{array}$ & Increase & Increase & $\begin{array}{l}\text { Large } \\
\text { Decrease }\end{array}$ & Degrade \\
\hline $\begin{array}{l}\text { Increasing } \\
\mathrm{K}_{\mathrm{D}}\end{array}$ & $\begin{array}{l}\text { Small } \\
\text { Decrease }\end{array}$ & Decrease & Decrease & $\begin{array}{l}\text { Minor } \\
\text { Change }\end{array}$ & Improve \\
\hline
\end{tabular}

A family of unit-step response curves with various values of damping coefficients, $\zeta$ is shown in fig. 3 . In control theory, a system with a damping coefficient of $\zeta=0$ is considered marginally stable and results in a sinusoidal error signal. A damping coefficient of $0<\zeta<1$, results in a system known as underdamped where the error signal exhibits a damped sunusoidal oscillation and at steady state, no error exists between input (target volume) and output (actual volume). A damping coefficient of $\zeta=1$ is considered critically damped (often the ideal situation) which exhibits the fastest response. A coefficient of $\zeta>1$ is known as an overdamped system and is sluggish in responding to any inputs.

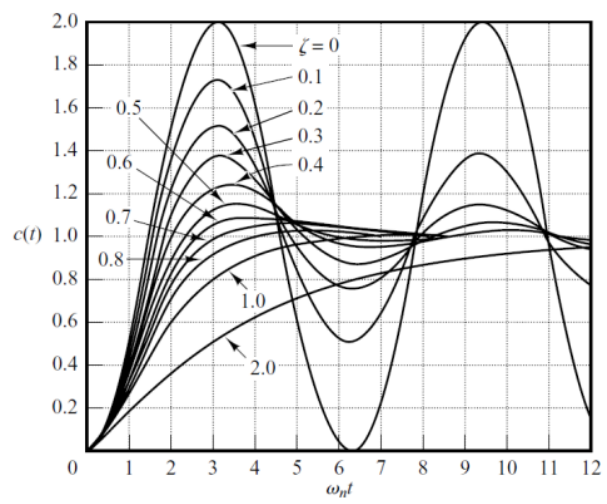

Figure 3. Unit-step response curves for a second order system with varying damping ratios [9]

\section{Methodology}

It is expected that, the smaller the value of the element removal ratio used, the more accurate is the final design, at the expense of larger computation time [10]. This philosophy is based on elemental removal ratio strategy considered with respect to the current number of elements. The motivation behind the applied control theory is to allow for large amounts of material to be removed at the beginning of the optimization when there exists a lot of inefficient material and for small amounts of material to be removed towards then end of the optimization as the volumetric error approaches zero.

The plot of volume versus iteration number can be generalized as a nonlinear function which starts at some arbitrary initial volume, then approaches and eventually reaches the prescribed target volume. Note that the traditional constant evolutionary rate results in a volume graph that resembles a piecewise linear function as shown in fig. 2 but the adaptive evolutionary rate results in a nonlinear graph as presented as seen in fig. 6 .

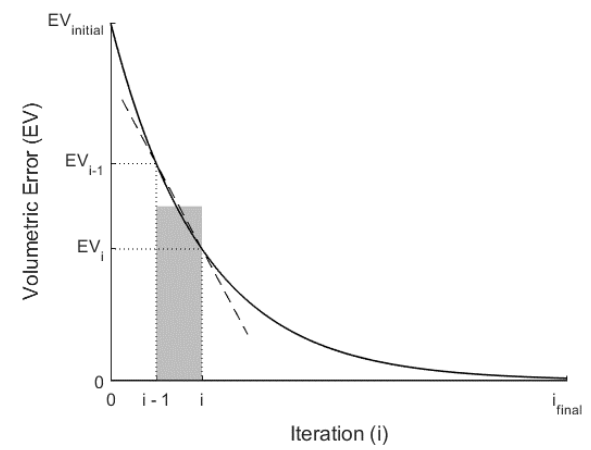

Figure 4. The volumetric error plot with a visualization of the volumetric error integral calculation (shaded grey box) and the volumetric error derivative (dashed line)

The volumetric error is calculated as the difference in the current structural volume and the target structural volume and demonstrated convergence is graphed in fig. 4. Volumetric Error $\left(\mathrm{E}_{\mathrm{v}}\right)$ is calculated as:

$$
E_{V_{i}}=V_{\text {target }}-V_{i}
$$

The volumetric integral error is caluclated to be the integral of the volumetric error signal which may be visualized as the area under the volumetric error graph as demonstrated in fig. 4. Volumetric Integral Error $\left(\int E_{D}\right)$ is calculated as:

$$
\int_{0}^{i} E_{v} \approx \int_{0}^{i-1} E_{v}+\frac{E_{v_{i}-1}+E_{v}}{2}(i-(i-1))
$$

Where $i$ denotes the current iteration number. Note that the (i - (i - 1))term of (8) evaluates to 1 , and therefore may be omitted during implementation.

The volumetric error derivative is calculated as the rate of change of the volumetric error. This can be simplified to represent the slope of the line passing through two adjacent points of the volumetric error graph as shown in the right graph of fig. 4. Volumetric Error Derivative $\left(E_{v}{ }^{\prime \prime}\right)$ is calculated as:

$$
\frac{d E_{V_{i}}}{d i} \approx \frac{E_{v_{i}}-E_{v_{i-1}}}{i-(i-1)}
$$

Similar to (9), the (i - (i - 1)) term of equation (8) evaluates to 1 , and therefore may be omitted during implementation.

Evolutionary Rate (ER):

$$
E R_{\mathrm{i}}=K_{P} E_{v_{\mathrm{i}}}+K_{I} \int_{0}^{\mathrm{i}} E_{\mathrm{V}}+K_{D} E_{v_{\mathrm{i}}}^{v}
$$

An alternative formula may be used for the next iterations target volume in lieu of (5). This method allows for the underdamped cases where the evolution's volume overshoots the target volume and returns to an equilibrium at the target volume. 


$$
V_{\mathrm{i}+1}=\min \left(\max \left(V_{\mathrm{i}}-E R_{v} 0\right), 1\right)
$$

Tuning a proportional-integral-derivative (PID) controller appears to be conceptually intuitive, but can be hard in practice, if multiple (and often conflicting) objectives such as short transient and high stability are to be achieved [11]. There exist many suggested methods for tuning a PID controller but the majority of them require increasing the proportional gain until the system becomes unstable or marginally unstable which breaks down the topology optimization algorithm when implemented.

For tuning the PID controller, the authors suggest starting with a derivative gain of zero and a very small value of the integral gain $\left(0.0001<\mathrm{k}_{\mathrm{I}}<0.001\right)$ slowly increasing the proportional gain until just after the algorithm has reached similar performance (e.g. similar compliance and settling time) as the standard algorithm. Then, the user should start increasing the derivative gain to make the volume convergence plot resemble that of a critically damped system. If the system seems to take too long to converge, the user should increase the integral gain slightly. The authors have find that the controller exhibits similar performance characteristics for varying mesh resolutions so it is suggested that the tuning be carried out on a low-resolution mesh then applied to the applicable high-resolution mesh.

\section{RESULTS AND DISCUSSION}

The first sample topology optimization with the PID controller presented in fig. 5 is to demonstrate how closely its volumetric convergence resembles that of the response of a second order linear dynamic system. With the gain tuning values of $K_{P}=0.2, K_{D}=0.3$ and $K_{I}=0.04$, it can be seen that at first the algorithm overshoots its target volume of $50 \%$ and reaches a volume fraction of $29 \%$, then overshoots the target again on its way back up and reaches a volume fraction of 55\% before eventually reaching its target and settling down. This behaviour resembles that of a system with a damping coefficient of 0.3 .

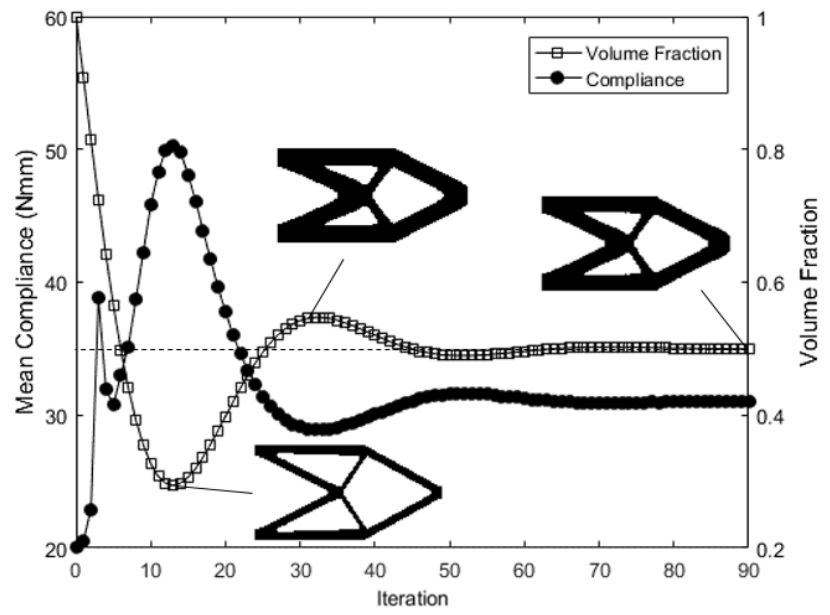

Figure 5. Topology optimization result with a PID tuning of $\mathrm{k}_{\mathrm{P}}=0.2, \mathrm{k}_{\mathrm{D}}=0.3$, $\mathrm{k}_{\mathrm{I}}=0.04$, resulting in a final compliance of $31.06 \mathrm{Nmm}$
The second sample optimization run aims to demonstrate similar performance to that of the standard BESO algorithm but with improved convergence characteristics. The example also uses the standard volume calculation of (5) instead of the new proposed volume calculation of (12) like the previous example. It can be seen that with a controller tuning of $\mathrm{k}_{\mathrm{P}}=0.2, \mathrm{k}_{\mathrm{D}}=0.3, \mathrm{k}_{\mathrm{I}}=0.04$, the optimization reaches a similar compliance within the same number of iterations. The volume convergence and incidentally the compliance make big changes at first when the volumetric error is the highest and small, gradual changes towards the end when the volumetric error is near zero.

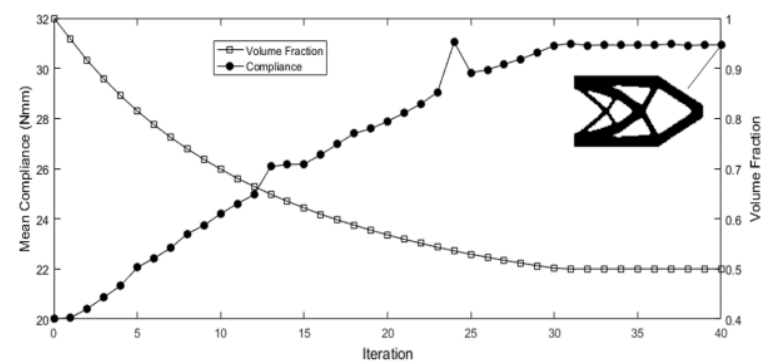

Figure 6. Topology optimization result with a PID tuning of: $\mathrm{k}_{\mathrm{P}}=0.125, \mathrm{k}_{\mathrm{D}}=$ $0.35, \mathrm{k}_{\mathrm{I}}=0.002$, resulting in a final compliance of $30.93 \mathrm{Nmm}$

The addition of the PID controller to the evolutionary rate of the BESO algorithm uses very little resources but provides the user with increased control on the algorithm's convergence as well as other surprising characteristics. The authors have found tunings that can achieve better compliance in less iterations than the standard BESO algorithm but the convergence plot is highly unstable. The authors have also found tunings that seem to provide length scale invariance regardless of the mesh resolution chosen. These intriguing cases will explored further in a future publication as the goal of this paper was to just introduce the topic.

Additionally, the evolutionary rate plays the similar role as the move limit in mathematical programming and the step size in optimality criteria methods [10]. This fact could be used to extend the PID control of the evolutionary rate of the BESO method to the move limit of the SIMP or MMA methods and will also be explored in future publications.

\section{CONCLUSION}

This paper presents an approach to control the convergence of Bi-Directional Evolutionary Structural Optimization algorithm. It is shown the evolutionary rate is an effective control parameter for a closed-loop Proportional-IntegralDerivative controller in the Bi-Directional Evolutionary Structural Optimization method. The developed methodology provides the user with effective control of various convergence characteristics of the volumetric evolution during the topology optimization process. 


\section{ACKNOWLEDGMENT}

The research support provided by the Natural Science and Engineering Research Council of Canada (NSERC) is greatly appreciated.

\section{REFERENCES}

[1] X. Huang and Y. Xie, Evolutionary topology optimization of continuum structures. Chichester, West Sussex, U.K: Wiley, 2010.

[2] G. Rozvany, "A critical review of established methods of structural topology optimization", Structural and Multidisciplinary Optimization, vol. 37, no. 3, pp. 217-237, 2008.

[3] F. Kaji and A. Barari, "Evaluation of the Surface Roughness of Additive Manufacturing Parts Based on the Modelling of Cusp Geometry", IFACPapersOnLine, vol. 48, no. 3, pp. 658-663, 2015.

[4] A. Barari, "Inspection of the machined surfaces using manufacturing data", Journal of Manufacturing Systems, vol. 32, no. 1, pp. 107-113, 2013.

[5] N. Siraskar, R. Paul and S. Anand, "Adaptive Slicing in Additive Manufacturing Process Using a Modified Boundary Octree Data
Structure", Journal of Manufacturing Science and Engineering, vol. 137, no. 1, p. 011007, 2014.

[6] H. Gohari and A. Barari, "A quick deviation zone fitting in coordinate metrology of NURBS surfaces using principle component analysis", Measurement, vol. 92, pp. 352-364, 2016.

[7] O. Querin, G. Steven and Y. Xie, "Evolutionary structural optimisation (ESO) using a bidirectional algorithm", Engineering Computations, vol. 15, no. 8, pp. 1031-1048, 1998.

[8] K. Åström and T. Hägglund, "The future of PID control", Control Engineering Practice, vol. 9, no. 11, pp. 1163-1175, 2001.

[9] K. Ogata, Modern control engineering, 5th ed. [Delhi]: Pearson, 2016.

[10] D. Nha Chu, Y. Xie, A. Hira and G. Steven, "On various aspects of evolutionary structural optimization for problems with stiffness constraints", Finite Elements in Analysis and Design, vol. 24, no. 4, pp. 197-212, 1997.

[11] Kiam Heong Ang, G. Chong and Yun Li, "PID control system analysis, design, and technology", IEEE Transactions on Control Systems Technology, vol. 13, no. 4, pp. 559-576, 2005. 